\title{
KÄHLERIAN THREE-MANIFOLD GROUPS
}

\author{
D. KOTSCHICK
}

\begin{abstract}
We prove that if the fundamental group of an arbitrary three-manifold not necessarily closed, nor orientable - is a Kähler group, then it is either finite or the fundamental group of a closed orientable surface.
\end{abstract}

\section{Introduction}

It has been well known for more than 20 years now that the fundamental groups of compact Kähler manifolds are in many ways very different from three-manifold groups. For example, cf. [2], Kähler groups are indecomposable under free products, and are far from real hyperbolic groups of dimension $\geq 3$, whereas the class of three-manifold groups is closed under taking free products and, according to Thurston, contains many hyperbolic groups of dimension 3. Nevertheless, it was only comparatively recently that Dimca and Suciu [5] proved the long-expected result that a Kähler group that is also the fundamental group of a closed three-manifold must be finite. In [13], I gave a simple proof of that result using essentially only the Albanese map and group cohomology.

The purpose of this paper is to give a variation of that proof which covers the fundamental groups of all three-manifolds, not only the closed ones. We shall prove:

Theorem 1. If the fundamental group of some three-manifold is infinite and a Kähler group, then it is the fundamental group of a closed orientable surface.

This generalizes both the theorem of Dimca-Suciu [5] and a very recent result of Friedl and Suciu [7], who considered compact three-manifolds with non-empty toroidal boundary. The problem of determining all Kählerian three-manifold groups was suggested by [7]. Discussing infinite groups only, as we do here, is an insignificant restriction, since all finite groups are in fact Kähler by a classical result of Serre. Furthermore, finite three-manifold groups are well understood.

The proof of [13] relied on Poincaré duality for closed manifolds and does not work in the general case. Still, we follow the same strategy as in that proof, looking at the homomorphism on fundamental groups induced by the Albanese map of a Kähler manifold and the naturality of the cup product in group cohomology.

The proof of Theorem 1 given here depends on modern developments in threemanifold topology only when dealing with closed manifolds with vanishing virtual first Betti numbers. The case of manifolds with non-empty boundary is more elementary, and requires only pre-Thurston results about three-manifolds that were available forty years ago.

Received by the editors January 7, 2013; (c) D. Kotschick 2013.

2000 Mathematics Subject Classification. primary 32Q15, 57M05, 57N10; secondary 14F35, $20 \mathrm{~F} 05$. 


\section{The proof}

Let $M$ be an arbitrary three-manifold with infinite fundamental group. Assuming that $\pi_{1}(M)$ is a Kähler group, we would like to prove that it is an orientable closed surface group. We do this by first going through a series of straightforward reduction steps, and then dealing with the crucial case of a compact aspherical three-manifold in Theorem 2 below.

2.1. Compactness. As usual, the term Kähler group denotes a group that is the fundamental group of some closed Kähler manifold. In particular, Kähler groups are finitely presentable. Now it is a result of Jaco [12] that if the fundamental group of a three-manifold is finitely presentable, then it is also the fundamental group of a compact three-manifold, possibly with boundary. Thus we may assume that $M$ is compact.

2.2. Primeness. We may assume that $M$ is prime, since otherwise its fundamental group would be a non-trivial free product. This is not possible, either by Gromov's theorem [9]; see also [2], or, granting residual finiteness of $\pi_{1}(M)$, by [13, Corollary 3.2], where I showed that a residually finite free product has a finite index subgroup with odd first Betti number.

2.3. Asphericity. By assumption, $\pi_{1}(M)$ is infinite. It cannot be virtually cyclic since it is assumed to be Kähler. Therefore, our prime $M$ is irreducible and aspherical by the sphere theorem, compare [15]. Thus $\pi_{1}(M)$ is a torsion-free group of cohomological dimension $\leq 3$.

2.4. Passage to finite coverings. Note that finite index subgroups of Kähler groups are trivially Kähler. Since a torsion-free group containing the fundamental group of a closed orientable surface as a finite index subgroup is itself a closed surface group, we may replace $M$ by an arbitrary finite covering, once we check that the fundamental groups of non-orientable surfaces are not Kähler. This is indeed so, since their first Betti numbers are positive $\left(\mathbb{R} P^{2}\right.$ has been excluded by the assumption that we have infinite groups) but the cup product from $H^{1}$ to $H^{2}$ is trivial, contradicting the Hard Lefschetz property. Replacing $M$ by a finite covering we may assume that it is orientable, so that its boundary is orientable as well.

2.5. Capping off spherical boundary components. Next, capping off an $S^{2}$ in the boundary of $M$ by a three-ball does not change the fundamental group, so we may assume that $M$ does not have spherical boundary components.

2.6. Positivity of the first Betti number. If $M$ has non-empty boundary, then, since the boundary is orientable and not spherical, the boundary has non-trivial first Betti number, and so does $M$ itself by the "half lives, half dies" argument.

When $M$ is closed, it can of course be a rational homology sphere. By Agol's recent resolution of the virtually Haken conjecture [1], $M$ has a finite covering with positive first Betti number. However, we do not need this recent result. As discussed in [13, p. 1085/86], if $M$ were closed, with zero virtual first Betti number, then $\pi_{1}(M)$ would not be Kähler, using Perelman's results and a theorem of Carlson and Toledo. 
2.7. The main argument. We have now explained that Theorem 1 follows from:

Theorem 2. Let $M$ be a compact aspherical three-manifold with $b_{1}(M)>0$. If $\pi_{1}(M)$ is the fundamental group of a closed Kähler manifold $X$, then the Albanese map of $X$ induces an isomorphism between $\pi_{1}(M)$ and the fundamental group of a closed orientable surface.

Of course $M$ must then have non-empty boundary. In fact, it is a classical result of Heil [10, Proposition 1] that $M$ is an interval bundle over a surface.

For the proof of Theorem 2 assume that $X$ is a closed Kähler manifold with $\pi_{1}(X)=$ $\pi_{1}(M)$. The assumption that the first Betti number is positive implies that $X$ has a non-constant Albanese map. Since the target of the Albanese is aspherical, the map factors up to homotopy through the classifying space of $\pi_{1}(X)$, which we may take to be $M$. But the cohomological dimension of $M$ is strictly less than 4 , and so the Albanese image of $X$ must be a complex curve, necessarily of positive genus $g$. By a standard argument, this implies that the Albanese image is smooth, and the Albanese map has connected fibers, compare [8, p. 289]. Therefore, we are in the situation of the following lemma:

Lemma 3 ([4]). Let $f: X \longrightarrow C_{g}$ be a surjective holomorphic map with connected fibers from a compact complex manifold to a curve of genus $g \geq 1$. By marking the critical values $p_{1}, \ldots, p_{k}$ of $f$ with suitable integral multiplicities $m_{i} \geq 1$, one can define the orbifold fundamental group $\pi_{1}^{\text {orb }}\left(C_{g}\right)$ of $C_{g}$ with respect to these multiplicities, so that one obtains a short exact sequence

$$
1 \longrightarrow K \longrightarrow \pi_{1}(X) \longrightarrow \pi_{1}^{\text {orb }}\left(C_{g}\right) \longrightarrow 1
$$

in which the kernel $K$ is finitely generated, since it is a quotient of the fundamental group of a regular fiber of $f$.

The point is that if there are multiplicities $m_{i} \geq 2$, then the orbifold fundamental group on the right is rather larger than the usual topological fundamental group of $C_{g}$, and this ensures that the kernel $K$ is finitely generated, compare the discussion in $[4,14,17]$. To prove Theorem 2 we only have to prove that $K$ is trivial, for then $\pi_{1}(M)=\pi_{1}(X)=\pi_{1}^{\text {orb }}\left(C_{g}\right)$. As $\pi_{1}(M)$ is torsion-free, the orbifold structure must be trivial, and $\pi_{1}^{\text {orb }}\left(C_{g}\right)$ is just the usual fundamental group of $C_{g}$.

By the solution of the Fenchel conjecture [3], $\pi_{1}^{\text {orb }}\left(C_{g}\right)$ has a surface group $\pi_{1}(S)$ as a finite index subgroup. So at the expense of replacing $M$ by a finite covering, compare 2.4, its fundamental group actually fits into the following extension:

$$
1 \longrightarrow K \longrightarrow \pi_{1}(M) \stackrel{\varphi}{\longrightarrow} \pi_{1}(S) \longrightarrow 1,
$$

with the same finitely generated $K$ as above. If $K=\operatorname{ker}(\varphi)$ is non-trivial, then by a result of Hempel and Jaco [11, Theorem 1] it is infinite cyclic. The five-term exact sequence of the extension (2.2) in real cohomology then reads

$$
0 \longrightarrow H^{1}(S ; \mathbb{R}) \stackrel{\varphi^{*}}{\longrightarrow} H^{1}(M ; \mathbb{R}) \longrightarrow H^{1}(K ; \mathbb{R})^{\pi_{1}(S)} \stackrel{\delta}{\longrightarrow} H^{2}(S ; \mathbb{R})=\mathbb{R} \stackrel{\varphi^{*}}{\longrightarrow} H^{2}(M ; \mathbb{R}) \text {. }
$$

If the connecting homomorphism $\delta$ is non-zero, then $H^{1}(M ; \mathbb{R})=H^{1}(S ; \mathbb{R})$, with identically zero cup product to $H^{2}(M ; \mathbb{R})$, since the cup product is natural under $\varphi^{*}$, which vanishes on $H^{2}(S ; \mathbb{R})$ by exactness. This contradicts the Kählerness of $\pi_{1}(M)$ via the Hard Lefschetz theorem. If $\delta$ is zero, then, after possibly passing to a double 
covering again to ensure that the action of $\pi_{1}(S)$ on $H^{1}(K ; \mathbb{R})=\mathbb{R}$ is trivial, we have $b_{1}(M)=1+b_{1}(S)$, which is odd, again contradicting the Kählerness of $\pi_{1}(M)$.

This completes the proof of Theorem 2 and therefore also of Theorem 1.

2.8. An alternative argument. The proof of Theorem 2 given above has the pleasant feature of dealing with the cases that $M$ is closed or with non-empty boundary uniformly. In particular, it gives yet another treatment of closed three-manifolds that is different from $[5,13]$.

Now, taking for granted the closed case, an alternative - and much more high-tech - treatment of manifolds with non-empty and non-spherical boundary is implicit in my recent paper [14], where I discussed Kähler groups of positive deficiency. The deficiency of a finitely presentable group is the maximum over all presentations of the difference of the number of generators and the number of relators. For a compact three-manifold $M$ with non-empty boundary Epstein [6, Lemma 2.2] proved that

$$
\operatorname{def}\left(\pi_{1}(M)\right) \geq 1-\chi(M)=1-\frac{1}{2} \chi(\partial M) .
$$

Since the Euler characteristic of the boundary is non-positive, the deficiency of $\pi_{1}(M)$ is positive. If $\partial M$ has at least one boundary component with negative Euler characteristic, then the deficiency is at least 2, and [14, Theorem 2] applies, to say that the kernel $K$ in (2.1) must be trivial and $\pi_{1}(M)$ is isomorphic to $\pi_{1}^{\text {orb }}\left(C_{g}\right)$. Since $\pi_{1}(M)$ is torsion-free, the orbifold structure must be trivial, and this is an ordinary surface group.

If $M$ has toroidal boundary, then the deficiency may well be $=1$. As explained in $[14$, p. 646], the results there go through for deficiency one groups whenever one knows that the kernel $K$ in (2.1) is not just finitely generated, but finitely presentable, or at least of type $F P_{2}$. This is the case here, since by a result of Scott [16] and Shalen (unpublished), $\pi_{1}(M)$ is coherent, meaning that any finitely generated subgroup must be finitely presentable. Thus, the results of [14] imply Theorem 2 above in all cases when $M$ has non-empty boundary.

The case of a closed $M$ cannot be dealt with by appealing to [14], since for a closed aspherical three-manifold the fundamental group has vanishing deficiency by another result of Epstein, see [6, Lemma 3.1].

\section{Acknowledgments}

Research done at the Institute for Advanced Study in Princeton with the support of The Fund For Math and The Oswald Veblen Fund.

\section{References}

[1] I. Agol, The virtual Haken conjecture, preprint 12 April 2012, arXiv:1204.2810v1 [math.GT].

[2] J. Amorós, M. Burger, K. Corlette and D. Kotschick and D. Toledo, Fundamental Groups of Compact Kähler Manifolds, Mathematical Surveys and Monographs, 44, Amer. Math. Soc., Providence, RI. 1996.

[3] S. Bundgaard and J. Nielsen, On normal subgroups with finite index in F-groups, Mat. Tidsskr. B. 1951 (1951), 56-58.

[4] F. Catanese, Fibred Kähler and quasi-projective groups, Adv. Geom. Special Issue (2003), suppl., S13-S27.

[5] A. Dimca and A.I. Suciu, Which 3-manifold groups are Kähler groups?, J. Eur. Math. Soc. 11 (2009), 521-528. 
[6] D.B.A. Epstein, Finite presentations of groups and 3-manifolds, Q. J. Math. Oxford 12 (1961), 205-212.

[7] S. Friedl and A.I. Suciu, Kähler groups, quasi-projective groups, and 3-manifold groups, preprint 30 December 2012, arXiv: 1212.3022v2 [math.GT].

[8] R. Friedman, Algebraic surfaces and holomorphic vector bundles, Springer Verlag, 1998.

[9] M. Gromov, Sur le groupe fondamental d'une variété kählérienne, C. R. Acad. Sci. Paris Sér. I Math. 308 (1989), 67-70.

[10] W. Heil, On the existence of incompressible surfaces in certain 3-manifolds. II, Proc. Amer. Math. Soc. 25 (1970), 429-432.

[11] J. Hempel and W. Jaco, 3-Manifolds which fiber over a surface, Amer. J. Math. 94 (1972), 189-205.

[12] W. Jaco, Finitely presented subgroups of three-manifold groups, Invent. math. 13 (1971), $335-346$.

[13] D. Kotschick, Three-manifolds and Kähler groups, Ann. Inst. Fourier 62 (2012), 1081-1090.

[14] D. Kotschick, The deficiencies of Kähler groups, J. Topology 5 (2012), 639-650.

[15] J.W. Milnor, A unique decomposition theorem for 3-manifolds, Amer. J. Math. 84 (1962), 1-7.

[16] G.P. Scott, Finitely generated 3-manifold groups are finitely presented, J. Lond. Math. Soc. 6 (1973), 437-440.

[17] C.T. Simpson, The ubiquity of variations of Hodge structure, in 'Complex geometry and Lie theory' (Sundance, UT, 1989), 329-348, Proc. Symp. Pure Math., 53, Amer. Math. Soc., Providence, RI, 1991.

Mathematisches Institut, LMU MünChen, Theresienstr. 3980333 MünChen, Germany

E-mail address: dieter@member.ams.org 
